\title{
ON-LINE CONTROL OF A FLEXIBLE BEAM USING ADAPTIVE FUZZY CONTROLLER AND PIEZOELECTRIC ACTUATORS
}

\author{
Gustavo Luiz C. M. de Abreu* \\ gmanhaes@mecanica.ufu.br
}

\author{
José F. Ribeiro* \\ jribeiro@mecanica.ufu.br
}

*Faculdade de Engenharia Mecânica, Universidade Federal de Uberlândia, Av. João Naves de Ávila, 2160,

Uberlândia, MG, 38400-902

\begin{abstract}
This paper presents a design of adaptive fuzzy controllers applied to the control of vibrations in flexible structures containing distributed piezoelectric actuator patches. The adaptive fuzzy controller is constructed from a set of fuzzy IF-THEN rules whose parameters are adjusted on-line according to some adaptation law. The adaptive law is derived based on the Lyapunov's stability theory. This control methodology is experimentally verified in a steel cantilever test beam and a set of experimental tests are made in the system to verify the efficiency of the adaptive fuzzy controller proposed.
\end{abstract}

KEYWORDS: Adaptive Fuzzy Controllers, Lyapunov's Stability Theory, Flexible Structures and Piezoelectric Actuators.

\section{INTRODUCTION}

The great progress experimented by the theory of the fuzzy controllers in the last years has been opening new possibilities of practical application for these controllers.

In the last two decades, the subject area of smart/intelligent materials and structures has experienced tremendous growth in terms of research and development (Crawley \& deLuis, 1987). One reason for this activity is that it may be possible to create certain

Artigo submetido em $12 / 6 / 2001$

1a. Revisão em 4/8/2003

Aceito sob recomendação do Ed. Assoc. Prof. Takashi Yoneyama types of structures and systems capable of adapting to or correcting for changing operating conditions. The advantage of incorporating these special types of materials into the structure is that the sensing and actuating mechanism becomes part of the structure by sensing and actuating strains directly. This is more known like piezoelectric phenomena, i.e., direct and converse piezoelectric effects. When a mechanical force is applied to a piezoelectric material, an electric voltage or charge will be generated. On the other hand, when an electric field is applied to the material, a mechanical force will be induced because of the converse piezoelectric effect. With the recent advances in piezoelectric technology, it has been shown that the piezoelectric actuators based on the converse piezoelectric effect can offer excellent potential for active vibration control techniques, especially for vibration suppression or isolation.

Recent research has focused on the applications of piezoelectric sensor and actuator in smart structures. Crawley \& de Luis (1987) were among the first to embed piezoelectric materials in composite laminated beams. Chen et al. (1996) and Lin et al. (1999) presented a formulation methodology to the control of vibrations in composite structures with bonded piezoelectric sensors and actuators. Vibration suppression of composite smart structures by using piezoelectric sensors and actuators were also analyzed numerically by Chou et al. (1997).

Effective applications in vibration control, however, require that the system dynamics can be adequately 
and/or accurately determined and that the controller design can be easily implemented. Vibration control of smart structures using fuzzy controllers has thus been receiving attention for their ability to deal with nonlinearities, uncertainties in terms of vagueness, ignorance, and imprecision, and provide a feasible alternative since they can easily capture qualitative aspects of human knowledge. Fuzzy controllers are most suitable for systems that cannot be precisely described by mathematical formulations. In this case, a control designer captures operators knowledge and converts it into a set of fuzzy control rules. The idea of the fuzzy logic is useful for representing linguistic terms numerically and making reliable decisions with ambiguous and imprecise events or facts. The benefit of the simple design procedure of a fuzzy controller leads to the successful applications of a variety of engineering systems (Lee, 1990). Zeinoun and Khorrami (1994) proposed a fuzzy logic algorithm for vibration suppression of a clamp-free beam with piezoelectric sensor/actuator, and Ofri et al. (1996) also used a control strategy based on Fuzzy Logic Theory for vibration damping of a large flexible space structure controlled by bonded piezoceramic actuators.

In this work, an adaptive fuzzy controller is constructed from a set of fuzzy IF-THEN rules whose parameters are adjusted on-line according to some adaptation law. The adaptive law is derived based on the Lyapunov's stability theory (Wang, 1992). The adaptive fuzzy controller is designed through the following steps: first, construct an initial controller based on linguistic descriptions (in the form of fuzzy IF-THEN rules) about the unknown plant from human experts; then, develop an adaptation law to adjust the parameters of the fuzzy controller on-line. We use the Sugeno-type of the fuzzy logic system to approximate the controller. Finally, the adaptive fuzzy controller is used to control the flexible beam with piezoelectric actuators.

The adaptive fuzzy controllers try to address this kind of problems for three requirements: a) for the first requirement, we use nonlinear adaptive control concepts, where nonlinearly is required in order to cover this systems type, and adaptively is required because the mathematical model of the bonding layer, which bonded the piezoelectrics to an elastic substructure, is unknown; b) for the second requirement, we use fuzzy systems as basic building blocks of the adaptive fuzzy controllers so that linguistic fuzzy IF-THEN rules can be directly incorporated into the controllers and c) for the third requirement, we use the Lyapunov synthesis approach to construct the basic adaptive fuzzy controllers.

This adaptive controller is experimentally verified in a steel cantilever test beam. A set of experimental tests is made in the system to verify the efficiency of the adaptive fuzzy controller proposed. Experimental results are shown, to support the effectiveness of the active vibration control, and, finally, a conclusion of this study is given.

\section{DESCRIPTION OF THE USED FUZZY LOGIC SYSTEM}

Fuzzy set theory was proposed by Zadeh (1965), and it was employed as an alternative to traditional modeling and control design in order to provide a suitable representation of complex systems. The basic configuration of the fuzzy logic systems considered in this paper is shown in Fig. 1.

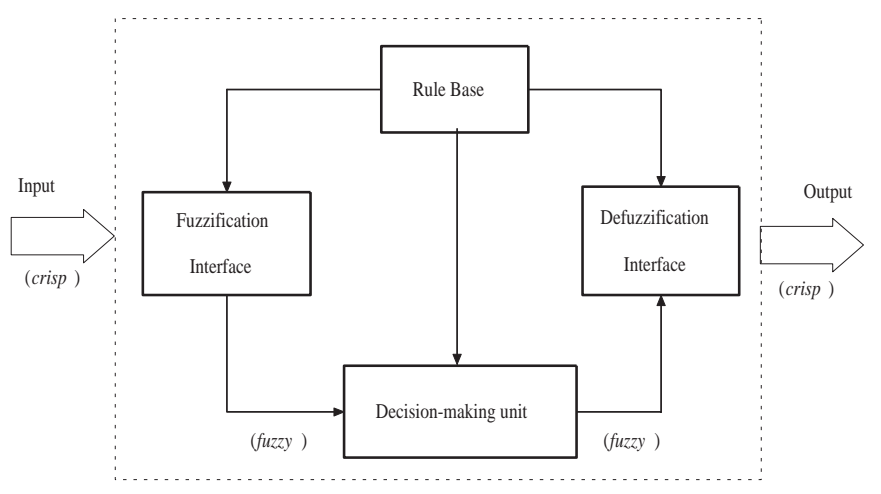

Figure 1: Basic Configuration of a fuzzy logic system.

In order to obtain the control design for a nonlinear or complex dynamic system, there are four basic steps in designing a conventional fuzzy logic controller (FLC) for a physical system: 1) the definition of input/output fuzzy variables; 2) the decision making of fuzzy control rules; 3) fuzzy inference logic, and 4) defuzzification and aggregation. The inference operations upon fuzzy if-then rules performed by fuzzy inference systems are described as follows.

The definition of input/output variables. The input/output variables of a fuzzy controller can be divided into system variables, and linguistic variables. Most fuzzy controllers employ the error and error rate of system variables as the input and the force, voltage or another variable of the control law as the output.

The fuzzy control rule is important to the successful operation of the fuzzy control system. The rule base (knowledge base), containing a number of fuzzy if-then 
rules, is composed as follows:

$$
\begin{aligned}
& R^{(m)}: I F x_{1} \text { is } A_{1}^{m} \text { and ... and } x_{n} \text { is } A_{n}^{m} \\
& \text { THEN } y=K_{m}
\end{aligned}
$$

where $x=\left(x_{1}, \ldots, x_{n}\right)^{T}$ and $y$ are the input and the output of the fuzzy logic system, respectively. $A_{i}^{m}$ is the label of the fuzzy set in $i$, for $m=1,2, \ldots, M$, and $K_{m}$ is the zero-order Sugeno parameter.

Fuzzy inference logic. The sup-algebraic product compositional rule of inference is employed in this work.

Defuzzyfication and aggregation. In order to obtain the correct control input for this control system, it is necessary to defuzzify the fuzzy sets and aggregate the qualified consequent parts to produce a crisp output. In this work, the mean of maximums method is employed to calculate the final output.

Based on these selections, the final output of the fuzzy controller can be calculated by:

$$
y=\frac{K_{1} \prod_{i=1}^{n} \mu_{A_{i}^{1}}\left(x_{i}\right)+\ldots+K_{m} \prod_{i=1}^{n} \mu_{A_{i}^{m}}\left(x_{i}\right)}{\prod_{i=1}^{n} \mu_{A_{i}^{1}}\left(x_{i}\right)+\ldots+\prod_{i=1}^{n} \mu_{A_{i}^{m}}\left(x_{i}\right)}
$$

If we constraint the denominator of the fuzzy system equation (eq. 2) to be equal to 2 by a dynamic scheme, we can rewrite equation 2 as:

$$
y(x)=\Theta^{T} \Xi(x)
$$

where:

$$
\Theta \triangleq\left[\begin{array}{llll}
K_{1} & K_{2} & \cdots & K_{m}
\end{array}\right]^{T}
$$

and

$$
\left.\Xi(x) \triangleq \frac{\left[\prod_{i=1}^{n} \mu_{A_{i}^{1}}\left(x_{i}\right) \quad \prod_{i=1}^{n} \mu_{A_{i}^{2}}\left(x_{i}\right) \quad \ldots \quad \prod_{i=1}^{n} \mu_{A_{i}^{m}}\left(x_{i}\right)\right.}{\prod^{n}}\right]
$$

Here, $\Theta$ represents the vector of adaptation parameters and $\Xi(x)$ vector of regressors.

\section{DERIVATION OF THE ADAPTIVE FUZZY CONTROLLER}

Consider the $n$ th-order nonlinear systems of the form

$$
\begin{aligned}
x^{(n)} & =f\left(x, \dot{x}, \ldots, x^{(n-1)}\right)+b u \\
y & =x
\end{aligned}
$$

where $f$ is an unknown but bounded continuous function, $b$ is a positive unknown constant, and $u$ and $y$ are the input and output of the system, respectively.

The control objective is to force $y$ to follow a given bounded reference signal $y_{m}$ under the constraints that all signals involved must be bounded. Hence, a feedback control $u$ based on fuzzy logic systems and an adaptive law for adjusting the parameters of the fuzzy logic systems are both determined to satisfy the following conditions:

- The closed-loop system must be globally stable and

- The tracking error $e=y_{m}-y$ should be as small as possible under the constraints in (4).

The design objective is to impose an adaptive fuzzy control algorithm so that the following asymptotically stable tracking:

$$
e^{(n)}+k_{1} e^{(n-1)}+\ldots+k_{n} e=0
$$

is achieved. The roots of polynomial in the characteristic equation of (5) are all in the open left-half plane via an adequate choice of coefficients $k_{1}, k_{2}, \ldots, k_{n}$.

We firstly show the design of an optimal control system based on the assumption that the system equations are completely known. The optimal control law is:

$$
u^{*}=\frac{1}{b}\left(-f(x)+\stackrel{(n)}{y}+k^{T} e\right)
$$

Since the roots $k_{1}, k_{2}, \ldots, k_{n}$ are in the left-half plane, the equation 5 implies that $\lim _{t \rightarrow \infty}(e(t))=0$. Now, since the system is unknown, we can not implement the optimal control law (eq. 6). We want to design an adaptive fuzzy control system to approximate this optimal control.

Let $u_{f}(x \mid \Theta)$ be an adaptive fuzzy control law in the form of:

$$
u_{f}(x \mid \Theta)=\Theta^{T} \Xi(x)
$$

Substituting $u_{f}(x \mid \Theta)$ into equation 4 we have:

$$
\dot{x}=f(x)+b u_{f}(x \mid \Theta)
$$

By adding and substracting $b u^{*}$ to equation 8 we obtain the error equation governing the closed-loop system:

$$
\dot{e}=\Lambda_{c} e+b_{c}\left(u^{*}-u_{f}(x \mid \Theta)\right)
$$


where

$$
\Lambda_{c}=\left[\begin{array}{ccccc}
0 & 1 & 0 & \ldots & 0 \\
0 & 0 & 1 & \ldots & 0 \\
\vdots & \vdots & \vdots & \ddots & \vdots \\
0 & 0 & 0 & \ldots & 1 \\
-k_{1} & -k_{2} & -k_{3} & \ldots & -k_{n-1}
\end{array}\right], b_{c}=\left[\begin{array}{c}
0 \\
0 \\
\vdots \\
0 \\
b
\end{array}\right]
$$

Now, define $V_{e}=\frac{1}{2} e^{T} P e$ where $P$ is a symmetric positive definite matrix satisfying the Lyapunov equation:

$$
\Lambda_{c}^{T} P+P \Lambda_{c}=-Q
$$

where $Q>0$. Using equation 11 and the error equation 9 , we have:

$$
\begin{aligned}
\dot{V}_{e} & =-\frac{1}{2} e^{T} Q e+e^{T} P b_{c}\left(u^{*}-u_{f}(x \mid \Theta)\right) \\
& \leqslant-\frac{1}{2} e^{T} Q e+\left|e^{T} P b_{c}\right|\left(\left|u^{*}\right|+\left|u_{f}(x \mid \Theta)\right|\right)
\end{aligned}
$$

Then, we replace the $u_{f}(x \mid \Theta)$ by the fuzzy inference system 7 and develop an adaptive law to adjust the parameter vector $\Theta$. We define the optimal parameter vector:

$$
\Theta^{*} \triangleq \arg \min _{\Theta}(\sup |\varepsilon|)
$$

where $\varepsilon$ is defined as $\varepsilon \triangleq u_{f}\left(x \mid \Theta^{*}\right)-u^{*}$, the control error. The error equation 9 can be rewritten as:

$$
\begin{aligned}
\dot{e} & =\Lambda_{c} e+b_{c}\left(u_{f}\left(x \mid \Theta^{*}\right)-u_{f}(x \mid \Theta)\right)-b_{c} \varepsilon \\
& =\Lambda_{c} e+b_{c} \Phi^{T} \Xi(x)-b_{c} \varepsilon
\end{aligned}
$$

where $\Phi \triangleq \Theta^{*}-\Theta$ and $\Xi(x)$ is the vector of regressors in equation 3. Now, define the Lyapunov function candidate:

$$
V=\frac{1}{2} e^{T} P e+\frac{b}{2 \gamma} \Phi^{T} \Phi
$$

where $\gamma$ is a positive constant. Using equations 14 and 11 we can derive:

$$
\dot{V}=-\frac{1}{2} e^{T} Q e+e^{T} P b_{c}\left(\Phi^{T} \Xi(x)-\varepsilon\right)+\frac{b}{\gamma} \Phi^{T} \dot{\Phi}
$$

Now, let $p_{n}$ be the last column of $P$, then from the equation 10 we have:

$$
e^{T} P b_{c}=e^{T} p_{n} b
$$

Substituting equation 16 into 15, we obtain:

$$
\dot{V}=-\frac{1}{2} e^{T} Q e+\frac{b}{\gamma} \Phi^{T}\left(\gamma e^{T} p_{n} \Xi(x)+\dot{\Phi}\right)-e^{T} P b_{c} \varepsilon
$$

If we choose the adaptive law as:

$$
\dot{\Theta}=\gamma e^{T} p_{n} \Xi(x)
$$

and using the fact that $\dot{\Phi}=-\dot{\Theta}$, equation 17 becomes:

$$
\dot{V} \leqslant-\frac{1}{2} e^{T} Q e-e^{T} P b_{c} \varepsilon
$$

which is the best we can achieve.

In summary, using the adaptation law given by eq. 18, we can guarantee that $\dot{V}<0$.

\section{IMPLEMENTATION OF THE ADAP- TIVE FUZZY CONTROLLER}

Briefly, the adaptive fuzzy controller is designed as follows:

1. Initialization phase: To ensure stability, fix the controller parameters $k$ and $Q$ as discussed earlier, and solve equation 11 to obatin $P>0$.

(a) Specify a set of fuzzy rules

(b) Set initial conditions (i.e. relative Sugeno parameters, $\Theta_{0}^{T}$ ).

2. Online phase:

(a) Adjust relative zero-order Sugeno parameters of rules by using the online adaptation algorithm of equation 18 in the following form:

$$
\Theta_{t+1}^{T}=\Theta_{t}^{T}+\gamma e^{T} p_{n} \Xi(x) d t
$$

where $t$ is the actual time and $d t$ is the sampling time.

Using equation 7 calculate the new control using the new parameter $\Theta_{t+1}^{T}$, as calculated in step above.

\section{CONTROL PROBLEM FORMULATION}

In this section, we apply the adaptive fuzzy controller to control a flexible structure using piezoelectric actuators.

An experimental apparatus was constructed and it is constituted by a flexible cantilever steel beam type structure with piezoelectric patches symmetrically bonded on both sides (see Fig. 2) to provide a bending movement in the structure and this system has been employed to illustrate the suggested adaptive control algorithm. 


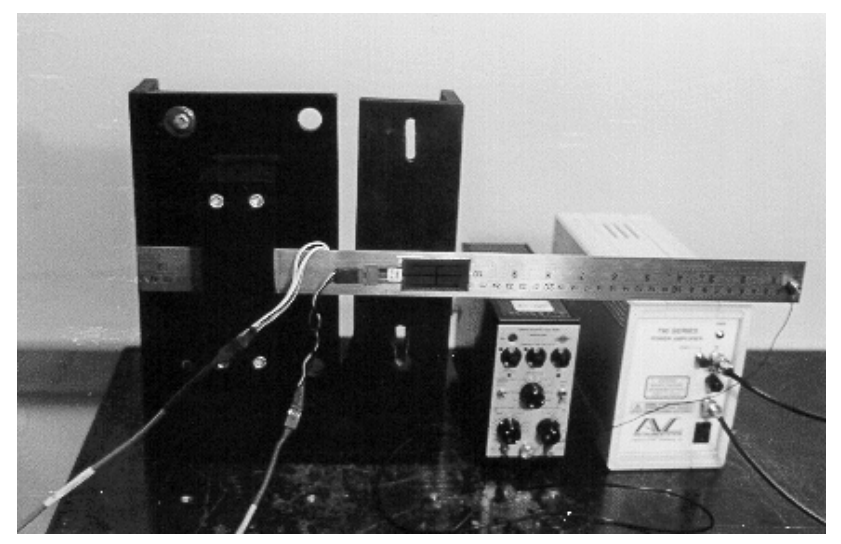

Figure 2: Experimental apparatus.

These piezoelectric elements are fed by a voltage amplifier (provide by company $A C X$ ), that amplifies the entrance voltage in the order $30 \mathrm{~V} / \mathrm{V}$.

The flexible structure which is $400[\mathrm{~mm}]$ long and has a cross-sectional dimensions of $34.5 \times 1.2[\mathrm{~mm}]$, contains two piezoelectric patches having a cross-sectional dimensions of $20.574 \times 0.254[\mathrm{~mm}]$ and its length is 46 $[\mathrm{mm}]$.

Each pair of piezoceramics (PZT) is bonded side by side to form two sets of actuators located 92.93 [mm] of the rigid support.

The schematic diagram for the vibration suppression experiment is shown in Figure 3. One accelerometer (B\&K 4375) attached at the tip through the charge amplifiers is used to measure the system displacement with integration circuits. Since the output voltage of the interface board is limited to $\pm 5[\mathrm{~V}]$, a voltage amplifier (ACX) is needed to drive the actuator in the range of $\pm 150[\mathrm{~V}]$ for effective control performance.

According the schematic diagram shown in Figure 3, the accelerometer signal is read by the A/D converter that communicates with a microcomputer (used in the implementation of a processing digital system using the adaptive fuzzy control algorithm). The sampling time used was 3 miliseconds.

For the verification of the adaptive fuzzy controller proposed we present a set of experimental tests for the system without control and controlled.

We set the reference $y_{r e f}=0$ and design the adaptive fuzzy controller:

1. Initialization phase:

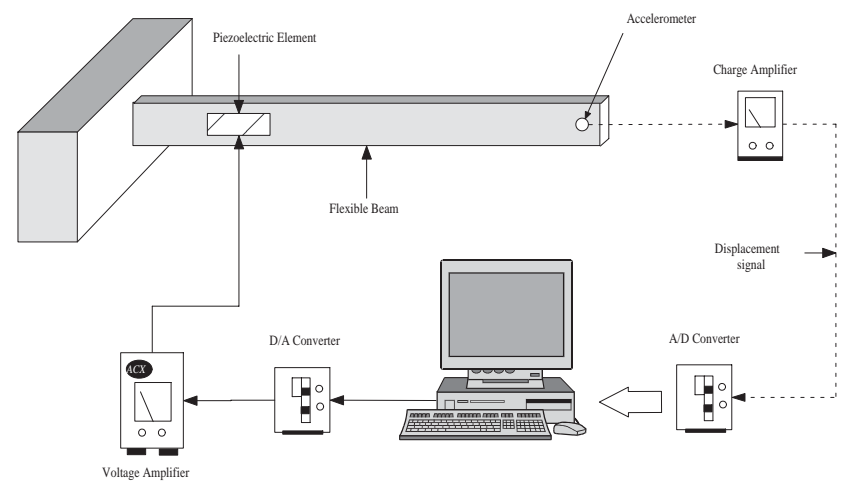

Figure 3: Schematic diagram of the vibration control experiment.

(a) We define $k=\left[\begin{array}{ll}3 & 2\end{array}\right], Q=\operatorname{diag}(5,5)$. Then, we solve (11) and obtain $P=$ $\left[\begin{array}{cc}5 & 2.5 \\ 2.5 & 2.5\end{array}\right]$.

(b) The fuzzy controller presents two inputs: error and change-of-error and one output: the output voltage applied to the voltage amplifier. We define membership functions (gaussshaped) of 3 fuzzy sets ( $N$ egative $-N$, Zero $Z$ and $P$ ositive $-P$ ) for the linguistic values of the input variables and the cross-point ratio of all is 0.5 . The ranges of the input variables are defined as: error: -1.5 to $1.5[\mathrm{~mm}]$ and change-of-error: -0.02 to $0.02[\mathrm{~mm} / \mathrm{s}]$.

The base of rules was composed by 9 rules and is given by Table 1 .

Table 1: Base of rules.

\begin{tabular}{|c|c|c|c|c|}
\cline { 3 - 5 } \multicolumn{2}{c|}{} & \multicolumn{3}{|c|}{ Change-of-error } \\
\cline { 3 - 5 } \multicolumn{2}{c|}{} & $N$ & $Z$ & $P$ \\
\hline \multirow{3}{*}{ Error } & $N$ & ${ }^{1} \boldsymbol{K}_{1}$ & ${ }^{2} \boldsymbol{K}_{2}$ & ${ }^{3} \boldsymbol{K}_{3}$ \\
\cline { 2 - 5 } & $Z$ & ${ }^{4} \boldsymbol{K}_{4}$ & ${ }^{5} \boldsymbol{K}_{5}$ & ${ }^{6} \boldsymbol{K}_{6}$ \\
\cline { 2 - 5 } & $P$ & ${ }^{7} \boldsymbol{K}_{7}$ & ${ }^{8} \boldsymbol{K}_{8}$ & ${ }^{9} \boldsymbol{K}_{9}$ \\
\hline
\end{tabular}

where $\left[\begin{array}{llll}K_{1} & K_{2} & \cdots & K_{9}\end{array}\right]^{T}$ is the vector of adaptation parameters.

Each one of the cells represents one rule constructed in the following form:

$R_{1}$ : If error is $N$ and change-of-error is $N$ then

output voltage is $\boldsymbol{K}_{1}$

(c) Set $\Theta_{0}^{T}=\left[\begin{array}{llll}0 & 0 & \cdots & 0\end{array}\right]^{T}$ and $\gamma=100$.

Online phase as described in section 4 .

2. To verify the controller efficiency, the initial excitation of the system is made by the actuators in 
Displacement (mm)

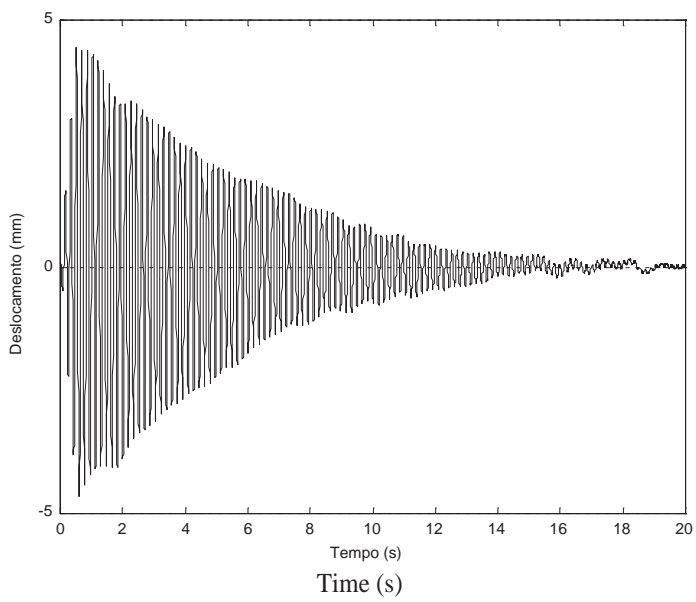

Figure 4: Experimental open-loop response.

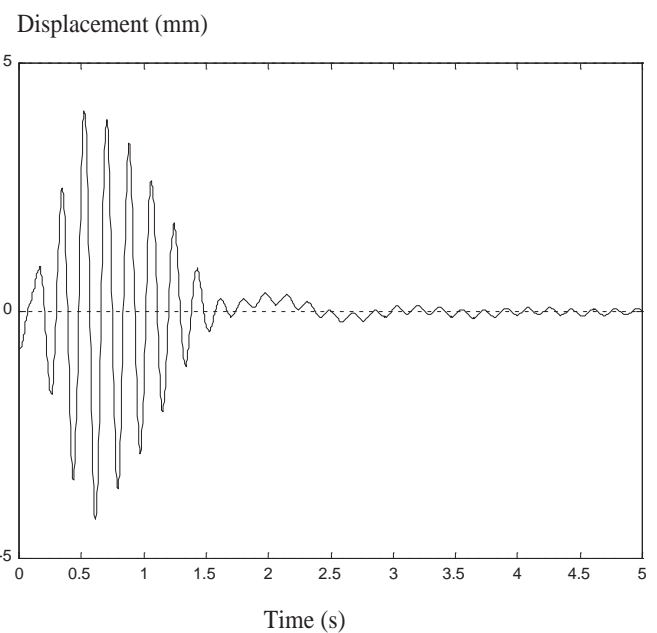

Figure 5: Experimental close-loop response.

the period of $0.5[\mathrm{~s}]$ and after this time they were utilized to control the flexible structure (see Fig. 6). The Figure 4 and 5 show the experimental open-loop response and close-loop response, respectively. The results clearly demonstrate the effectiveness of the adaptive fuzzy controller approach which achieve lower settling time.

The Figures 6 and 7 show the temporal evolution of output voltage applied to voltage amplifier and Sugeno parameters, respectively. The Sugeno parameters achieved are given in Table 2.
Output Voltage (V)

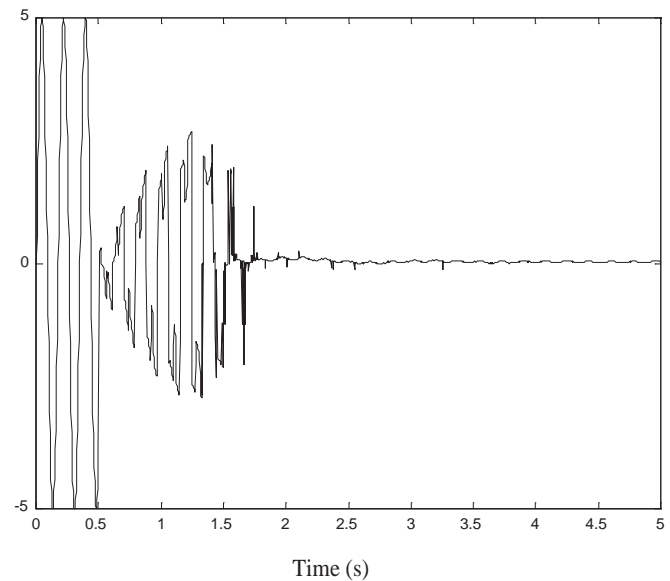

Figure 6: Output voltage response applied to voltage amplifier.

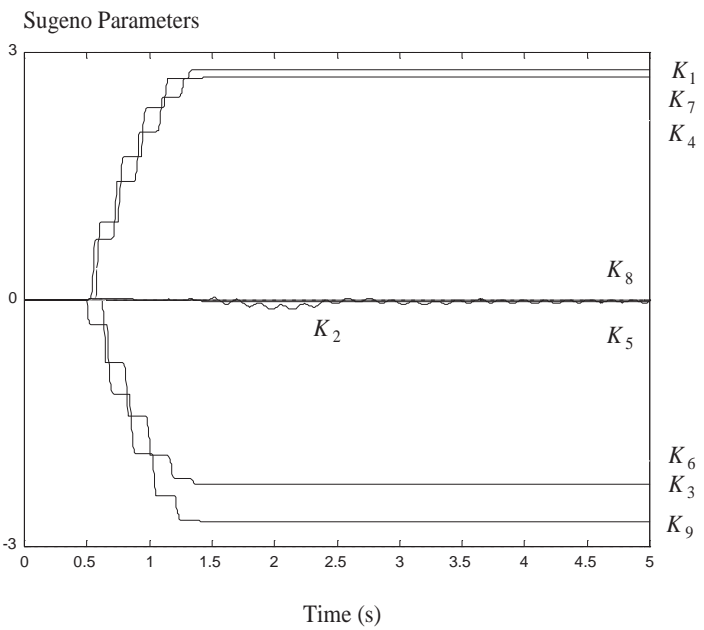

Figure 7: Temporal evolution of Sugeno parameters.

\section{CONCLUSIONS}

An adaptive fuzzy controller implemented in real-time was developed to control the vibrations of the flexible beam type structure using piezoelectric actuators. The results were presented for a simply cantilever steel flex-

Table 2: Final base of rules.

\begin{tabular}{|c|c|c|c|c|}
\hline & \multicolumn{3}{|c|}{ Change-of-error } \\
\hline & & $N$ & $Z$ & $P$ \\
\hline \multirow{3}{*}{ Error } & $N$ & 2,787 & $-0,003$ & $-2,247$ \\
\hline & $Z$ & 2,167 & 0,050 & $-1,960$ \\
\hline & $P$ & 2,708 & $-0,025$ & $-2,709$ \\
\hline
\end{tabular}


ible beam. By using a Lyapunov synthesis approach we expect to synthesise a stable adaptive system. The experimental results presented show that the present control methodology is effective and the control behaviors exhibit our predicted characteristics. From the experimental test results, it is concluded that the proposed design achieves the desired results.

The experimental results have shown that piezoceramic actuators bonded on a beam control efficiently the vibrations of this flexible structure. Work is in progress to use piezoelectric PVDF film materials as displacement sensors instead accelerometer.

\section{REFERENCES}

$A C X$, Active $C$ ontrol eXperts, Inc. All rights reserved, http://www.acx.com.

Chen, Chang-qing; Wang, Xiao-ming, and Shen, Yapeng (1996). Finite Element Approach of Vibration Control Using Self-Sensing Piezoelectric Actuators, Computers 85 Structures, Vol. 60, No. 3, pp. 505512.

Chou, Jyh-Horng; Chen, Shinn-Horng; Chang, MinYung, and Pan, An-Jia (1997). Active Robust Vibration Control of Flexible Composite Beams with Parameter Pertubations, International Journal of Mechanical Science, Vol. 39, No. 7, pp. 751-760.

Crawley, E. F., and De Luis, J. (1987). Use of Piezoelectric Actuators as Elements of Intelligent Structures, AIAA Journal, Vol. 25, No. 10, pp. 1373-1385.

Lee, M. (1990). Fuzzy Logic in Control Systems: Fuzzy Logic Controller - Part I and II, IEEE Transactions on Systems, Man and Cybernetics, Vol. 20, No. 2, pp. 404-435.

Lin, Chien-Chang, and Huang, Huang-Nan (1999). Vibration Control of Beam-Plates with Bonded Piezoelectric Sensors and Actuators, Computers and Structures, Vol. 73, pp. 239-248.

Ofri, A.; Tanchum, W., and Guterman, H. (1996). Active Control for Large Space Structure by Fuzzy Logic Controllers, IEEE, pp. 515-518.

Zadeh, L. A. (1965). Information and Control, Vol. 8, pp. 338-353.

Zeinoun, I. J., and Khorrami, F. (1994). An Adaptive Control Scheme Based on Fuzzy Logic and its Application to Smart Structures, Smart Mater. Struc., Vol. 3, pp. 266-276.
Wang, Li-Xin (1992). Stable Adaptive Fuzzy Control of Nonlinear Systems, Proceedings of the $31^{\text {st }}$ Conference on Decision and Control, Tucson, Arizona, December, pp. 2511-2516. 